\title{
Reducing Operating Room Costs through Real Time Cost Information Feedback: A Pilot Study
}

Christian H Tabib, Clinton D Bahler, Thomas J Hardacker, Kevin M Ball, Chandru P Sundaram

Department of Urology, Indiana University, Indianapolis, IN, USA

Running Title: Reducing costs with feedback reports

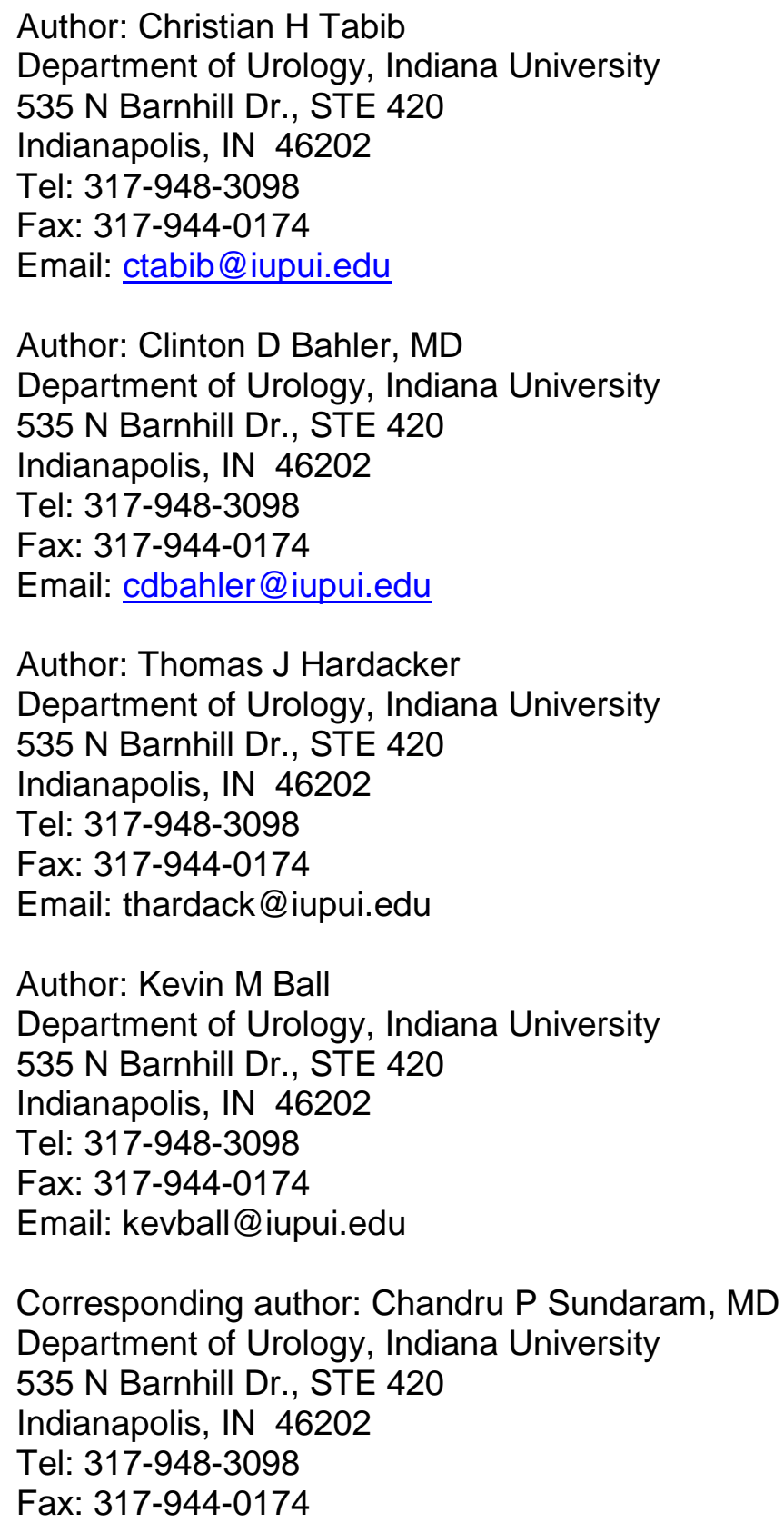

This is the author's manuscript of the article published in final edited form as:

Tabib, C. H., Bahler, C. D., Hardacker, T. J., Ball, K. M., \& Sundaram, C. P. (2015). Reducing Operating Room Costs Through Real-Time Cost Information Feedback: A Pilot Study. Journal of Endourology, 29(8), 963-968. http://doi.org/10.1089/end.2014.0858 
Email: sundaram@iupui.edu

\begin{abstract}
Objective: To create a protocol for providing real time operating room cost feedback to surgeons. We hypothesize that this protocol will reduce costs in a responsible way without sacrificing quality of care.
\end{abstract}

Methods: All operating room costs were obtained and recorded for robotic partial nephrectomy and laparoscopic donor nephrectomy. Prior to the beginning of this project, costs pertaining to the 20 most recent cases were analyzed. Items were identified from previous cases as modifiable for replacement or omission. Timely feedback of total OR costs and cost of each item used was provided to the surgeon after each case and costs were analyzed.

Results: A cost analysis of the robotic partial nephrectomy prior to the washout period indicates expenditures of \$5,243.04 per case. Ten recommended modifiable items were found to have an average per case cost of $\$ 1,229.33$ representing $23.4 \%$ of the total cost. A post washout period cost analysis found the total OR cost decreased by $\$ 899.67$ (17.2\%) due to changes directly related to the modifiable items. Therefore, $73.2 \%$ of the possible identified savings was realized. The same step-wise approach was applied to laparoscopic donor nephrectomies. The average total cost per case prior to the washout period was $\$ 3,530.05$ with $\$ 457.54$ attributed to modifiable items. After the washout period, modifiable items costs were reduced by $\$ 289.73$ (8.0\%). No complications occurred in the donor nephrectomy cases while one post-operative complication occurred in the partial nephrectomy group.

Conclusion: Providing surgeons with feedback related to operating room costs may lead to a change in surgeon behavior and decreased overall costs. Further studies are needed to show equivalence in patient outcomes. 


\section{Introduction}

The United States healthcare system is one of the most complicated and expensive systems in the world. In 2009 healthcare spending totaled \$2.5 trillion, which is equal to $17 \%$ of the United States’ 2009 Gross Domestic Product (GDP) of \$14.5 trillion. ${ }^{1}$ The Institute of Medicine has published a report detailing the necessity of cost reduction in order to maintain sustainability of the healthcare industry. In the report, they estimate that $\$ 765$ billion of the $\$ 2.5$ trillion total spending is spent on healthcare waste. ${ }^{2}$ Using these figures, healthcare waste accounts for over $5 \%$ of the U.S. GDP. The report examines categories where spending is wasted, which they identify to be missed prevention, unnecessary services, fraud, excessive administration services, inefficiently delivered services, and overpricing. It would be impossible to address all of this wasted spending at one time. Instead, it will be important to identify the root causes and determine a systemic solution to each.

The aim of this study was to increase physician awareness with regard to cost data in surgical procedures and develop an optimized protocol for implementing cost-saving measures. In doing so, it was the overall goal of the study to demonstrate that this could lead to behavioral change and cost savings. Providing physicians with comprehensive and real-time financial tracking data will allow for more in-depth self-evaluation and process improvement in the future. In order to do so safely and without sacrificing clinical outcomes, surgeons must be at the forefront of this evaluation. 


\section{Materials and Methods}

A linear stepwise process was utilized for identifying potential surgical waste and tracking outcomes as a result of increased pricing transparency (Figure 1). The method begins with the selection of a surgeon(s) and a specific surgical case. A retrospective cost analysis of the chosen case is to be undertaken next, so as to determine an average total cost for the procedure. Next, in consultation with an analysis team-in this case comprised of an OR nurse coordinator and surgeons who perform the case-modifiable items can be determined. These items should be those that potentially can be substituted for a lower-cost alternative or eliminated without compromising clinical outcomes. Once these items have been discussed with the primary surgeon an adequate "washout” period of five cases is allotted; which allows the surgeon to properly assess whether these changes are feasible. Following this, costs are tracked in a prospective fashion and compared to those from the retrospective analysis.

One surgeon was involved in this pilot study. Based on the surgeon's practice two frequently performed surgeries were chosen: laparoscopic robot assisted partial nephrectomy (LRAPN) and laparoscopic donor nephrectomy (LDN). LRAPNs performed by this surgeon were analyzed in both a retrospective and prospective fashion. Twenty cases were analyzed in a retrospective fashion prior to any intervention to calculate total costs for the cases. Item and cost data were gathered from SurgiNet ${ }^{\circledR}$ (Cerner Corp., Kansas City, MO) and the Lawson Portal (Infor, New York City, NY). This data was then compiled into a single spreadsheet for analysis

(Figure 2). In consultation with the attending surgeon, minimally invasive urology fellow, and the OR nurse coordinator, each item was assigned to one of five categories: Modifiable, robotic 
system costs, Suture, OR Time, Suction/Other. Following this consultation a five case "washout" period was observed prior to providing surgical cost feedback and tracking individual procedure costs.

For the post-intervention analysis, a cost feedback report was created using the data contained in the itemized cost spreadsheet. This was provided to the surgeon following each case. The cost feedback report provided information on average case cost, cost specific to the recent surgery, and usage/cost info regarding the selected modifiable items. A graphical representation of the categorical costs and the total Modifiable Cost for the procedure was illustrated on this final cost sheet (Figure 3). This data was compared against the previously generated average for the procedure. Following the analysis of LRAPNs, the same analysis was performed on LDNs by the same surgeon. Twenty LDN cases were reviewed in a retrospective manner. Total cost was estimated to be $\$ 3,530.05$ per case. The same attending, fellow, and OR nurse coordinator were consulted during the review of surgical items and their specific costs. Similar spreadsheets were created from the data. However, a new set of modifiable items were selected, specific for this surgical procedure with an average total cost of $\$ 457.54$ (13\% of total costs). Cost tracking and analysis were done in a prospective fashion following the five-case "washout" period.

Statistical analyses were performed using the Statistical Package for Social Sciences software (SPSS) version 22. We used an independent sample's T-test to compare the means of total costs and total Modifiable items cost savings before and after feedback. 


\section{Results}

Laparoscopic Robot-Assisted Partial Nephrectomies

Average total operating room (OR) cost prior to any intervention was found to be $\$ 5,243.04$ for LRAPNs. The average cost for items identified as Modifiable was $\$ 1,229.33$. This represented approximately $23.4 \%$ of the total OR cost. Following intervention, average total cost due to the discussed changes decreased by $\$ 899.67-17.2 \%$ of the pre-intervention total OR cost (Table 1 ). The total cost for all individual cases was tracked and compared with pre-intervention costs (Figure 4). All cost reduction indicated here resulted from changes directly related to modifiable items. This surgeon performs approximately 60 LRAPNs per year, yielding an estimated \$53,980.20 in annual cost savings. No intraoperative complications were observed. One major postoperative Clavien 3 complication occurred in which a patient had a pseudoaneurysm treated with IR embolization.

\section{Laparoscopic Donor Nephrectomies}

On average, a LDN generated \$3,530.05 in total OR cost. Modifiable items identified through consultation represented $\$ 457.54$ (13\%) of total cost. Following consultation and intervention, an average savings of $\$ 289.73$ per case $-8.2 \%$ - was realized. The total cost of each individual case was tracked and compared with the pre-intervention cases (Figure 4). Performing approximately 40 such cases per year, this surgeon could potentially save $\$ 11,589.20$ annually. No intraoperative or major postoperative complications were observed.

\section{Discussion}


The healthcare industry is rapidly progressing toward a model in which physicians will be asked to decrease costs without sacrificing clinical outcomes. A great deal of this cost-cutting will focus on operating room (OR) expenditures, as OR costs represent a large amount of hospital spending ${ }^{3}$, as well as potential profit ${ }^{4}$. Furthermore, it has been shown that a wide variability in both surgical cost and volatility of cost can exist between surgeons in the same hospital setting ${ }^{5}$. In order to ensure that healthcare professionals are driving these changes, it is important for physicians to understand how best to deliver healthcare value to patients ${ }^{6}$. In order for these cost savings to be instituted safely and effectively, it will be vital for surgeons to have access to realtime cost data, with the ability for quick implementation of changes.

In this study, inefficiently-delivered and unnecessary services in the operating room (OR) setting are the main points of emphasis. The total cost of any surgical procedure is largely dependent on the instrumentation and equipment used. Identifying and eliminating surgical equipment waste in the OR, while maintaining clinical outcomes, is a potential source of significant cost savings. Reducing OR excess is not a novel approach. A collaborative care approach was able to reduce hospital costs between 31\%-65\% for radical prostatectomy, with a large portion of those savings resulting from a shortening of surgical time and a reduction in use of surgical supplies. ${ }^{7}$ This approach is dependent on a large group of physicians reaching a consensus on best practice and relies on the physicians to alter behavior in order to realize these savings in the future. This approach, however, may not address the underlying factors as to why waste occurs. In order to identify and address wasted spending in the OR, it is important to examine these underlying factors leading to excess spending. The first barrier to saving is the medical device market. The medical device market totaled $\$ 153$ billion in 2008 and continues to grow. ${ }^{1}$ It is a large and complex industry that is not set up to reduce costs for hospitals. One 
reason for this is the widespread lack of transparency of equipment and instrumentation costs. Similarly, industry practices_-such as the use of rebates and bundled services — further complicate true cost identification. As such, hospital systems rarely pay full list price for the equipment they are sold. The continual research and development of these products serves to further increase equipment sale prices.

While the device market poses certain challenges, there are separate barriers for physicians who are attempting to reduce OR waste as well. Confidentiality clauses pertaining to equipment pricing is one such issue. The purchasers, most often hospital systems, are often contractually prohibited from sharing equipment cost data with third parties_-including physicians_-as device companies claim that pricing structures are trade secrets. One other issue that must be addressed is physician insensitivity. As it stands currently, there is little to no incentive for physicians to reduce overall cost. Without paying close attention to this cost, many surgeons are unknowingly and unnecessarily accumulating increased charges in the OR. As surgical procedures generate high revenues even in the presence of such wasted spending, hospital systems are unlikely to disrupt the practices of these physicians.

It is our contention that elevated surgical equipment costs are not due to surgeon indifference, but rather to a lack of transparency and accessibility. These issues are further exacerbated by non-disclosure agreements as previously mentioned. Furthermore, there is to date, a lack of robust analytical software capable of presenting these data effectively to physicians. These factors collectively serve to deter physicians from analyzing financial data and potentially reining in surgical costs. In order for financial changes to be instituted safely, surgeons must be engaged in the decision-making. In order to do so, there must be cost transparency and a means of presenting these data to surgeons in a concise and timely manner. 
In this study, transparency and effective presentation of surgical equipment financial data were shown to change surgeon behavior and decrease overall surgical cost as they related to LRAPNs (\$899.67 savings per case, $\mathrm{p}<0.001$ ) and LDNs ( $\$ 289.73$ savings per case, $\mathrm{p}<0.001)$. In this case, it was our finding that surgical equipment waste in the OR can be partially attributed to a lack of cost transparency and surgeon awareness of equipment pricing. The most striking example of the former was seen in the use of hemostatic agents containing thrombin. In LRAPNs, these products were used to achieve hemostasis in nearly every case prior to equipment analysis. It was not until the surgeon was made aware of the price of each, that he opted to discontinue their routine use, since there was no evidence that these agents were routinely essential.

Another example of utilization change involved the use of energy devices. Prior to cost feedback, partial nephrectomies began with pure laparoscopic dissection using a Harmonic Scalpel (Ethicon Endo-Surgery, Inc., Blue Ash, OH). However, after feedback it was decided to utilize only robotic energy devices. The surgeon favored a cost effective approach, which differed from his previous technique, to eliminate this item without sacrificing quality care. Additionally, once the surgeon became aware of the difference in cost between a staple reload and a new stapler, a plan was put forth to increase nurse training to increase utilization of staple reloads. In order to ensure quality, ischemia time must be minimized. However, the ability to reload the stapler in order to quickly and safely staple the renal vessels varies between OR nurses. If a nurse does not feel comfortable quickly reloading the stapler, then a new stapler would be used instead. The difference in cost is $\$ 191.70$ if a reload is used rather than a new stapler. This will result in cost savings while also ensure patient safety and quality outcomes in terms of minimizing ischemia times. Just as the aforementioned examples provided immediate and substantial cost decreases through behavior changes, the majority of financial savings in the 
OR will come from previously unknown or unidentified waste. Further analyses should identify expensive items that can be safely substituted for lower cost items. If physician incentives are used, they should be carefully structured to not only promote cost reduction but also patient safety.

The limitations of the study are primarily due to the lack of true financial data. While we were able to get exact costs of each item listed in the catalogue, we were unable to obtain the discounts allotted to most health care systems. However, we believe the comparison in percentage decreases rather than absolute decreases in price can still provide a relative idea of the true cost savings. Another limitation is the small sample size. Because this was an initial pilot program, we only worked with one surgeon on two low volume cases. In order to make a larger impact, high volume cases with multiple surgeons would be needed to see how much of an impact cost transparency has on surgeon behavior. Also, as this was a single surgeon study, we were unable to evaluate whether multiple surgeons could work together to eliminate duplication of supplies and equipment.

\section{Conclusions}

Though we were able to identify modifiable items—equipment that could be further analyzed to determine whether a cost effective alternative exists—it will be important to leave this final decision in the hands of each respective surgeon. Collaboration between surgeons performing the same procedure in the same hospital can result in use of standardized cost effective case carts and instrumentation, which will reduce costs on a wider scale. It is conceivable that cost savings can be identified through computer-generated metrics. However, the implementation could lead 
to suboptimal clinical outcomes, as the surgeon may not be able to provide adequate surgical care with the substitute. It is for this reason that we contend decreasing surgical costs will rely on the transparency and effective presentation of surgical cost data to surgeons, such that these decisions be entrusted to and made by the clinicians themselves.

In this study, it has been shown that cost transparency can affect behavior. As it stands currently, data was extracted by hand from two separate electronic databases and manually compiled into a useful final cost report. Clearly, more robust analytical software is necessary to provide surgeons with real-time cost tracking information ultimately needed to institute change. Furthermore, it will be vital to have effective lines of communication between surgeons and individuals along the supply chain line to reduce many aspects that contribute to OR waste. In order to maximize the cost-cutting measures that lie ahead, surgeons must be actively involved in clinical and financial decision-making to ensure that patient outcomes continue to be optimized along with cost reduction.

\section{Acknowledgements}

Nancy M Glenn, RN is the urology specialty coordinator for the IU-Health University Hospital. She provided item specific cost data in real-time along with countless insights into supply utilization. She also implemented changes and initiated operating room staff training.

\section{Author Disclosure Statement}

No competing financial interests exist. 


\section{References}

1. Yong PL, Saunders RS, Olsen L: The Healthcare Imperative: Lowering Costs and Improving Outcomes: Workshop Series Summary. Washington DC: The National Academies Press, 2011, pp175-218.

2. $\quad$ Federal Reserve Economic Data, Federal Reserve Bank of St. Louis 2014.

3. Elixhauser A, Andrews RM et al. Profile of Inpatient Operating Room Procedures in US Hospitals in 2007. Archives of Surgery 2010; 145: 1201-1208.

4. Rohloff $\mathrm{R}$, Holoska $\mathrm{M}$ et al. The greatest defense is a good offence. Surgery and ORs are a serious revenue stream for hospitals. Smart ones can enhance their financials by stretching IT into OR materials management. Health Management Technology 2005; 26: 32-33.

5. Hall BL, Campbell DA, Phillips LR, Hamilton BH, et al. Evaluating Individual Surgeons Based on Total Hospital Costs: Evidence for Variation in both Total Costs and Volatility of Costs. Journal of the American College of Surgeons 2006; 202: 565-576.

6. Porter ME, Teisberg EO, et al. How Physicians Can Change the Future of Health Care. JAMA 2007; 297: 1103-1111.

7. Koch MO, et al. Cost-Efficient Radical Prostatectomy. Seminars in Urologic Oncology 1995; 13: 197-203 
